\title{
Diffuse Lewy body disease presenting with a supranuclear gaze palsy
}

\author{
J M Fearnley, T Revesz, D J Brooks, R S J Frackowiak, A J Lees
}

National Hospital for Nervous Diseases, Queen Square, London

J M Fearnley

T Revesz

D J Brooks

RSJ Frackowiak

A J Lees

Correspondence to Dr Lees, National Hospital for Nervous Diseases, Queen Square, London WCIN 3BG, UK

Received 12 February 1990 and in final revised form 8 June 1990.

Accepted 6 July 1990

\begin{abstract}
A patient with diffuse Lewy body disease presented with supranuclear vertical and horizontal ophthalmoplegia, dementia, axial rigidity and falls, bradykinesia and pyramidal signs. This broadens the clinical presentation of this pathological diagnosis and re-emphasises the heterogeneity of patients diagnosed clinically as progressive supranuclear palsy (SteeleRichardson-Olszewski syndrome).
\end{abstract}

Diffuse Lewy body disease (DLBD) ${ }^{1}$ is clinically characterised by the combination of Parkinsonism, dementia and psychiatric symptoms. ${ }^{2}$ At presentation, however, the patient usually has only one of these symptoms making a definite clinical diagnosis impossible. Even at necropsy, the diagnosis is often overlooked, because of the difficulty in visualising cortical Lewy bodies on routine stains and the presence of Alzheimer changes, especially neuritic plaques, sometimes considered to be secondary to DLBD. ${ }^{3}$ The combination of Parkinsonism and dementia is not exclusive to DLBD and may occur in the Steele-Richardson-Olszewski syndrome (SRO), which is characterised by supranuclear ophthalmoplegia, pseudobulbar palsy, axial dystonia in extension, dementia and bradykinesia. ${ }^{45}$ Early in the disease only one component may be prominent, but most would agree that for a diagnosis to be at all certain a supranuclear downgaze palsy is essential. We describe a case of DLBD, which presented like SRO with Parkinsonism, dementia, ophthalmoplegia, dysarthria, axial rigidity, pyramidal signs and failure to respond to levodopa.

rigidity, hypomimia and in the limbs he had cogwheel rigidity, bradykinesia and an occasional rest tremor with a left sided preponderance. The tendon reflexes were brisk in the legs with bilateral Babinski signs. There was a disturbance of recent memory and features suggesting a temporoparietal defect: nominal dysphasia, dyscalculia, occasional right-left disorientation and mild constructional dyspraxia.

On formal psychometry, however, there was cognitive deterioration affecting a wide range of skills including tests sensitive to frontal lobe dysfunction. His verbal IQ was 99 (WAIS) compared with a premorbid IQ of 115 (National Adult Reading Test). CT scanning revealed generalised cortical atrophy. There was no response to a trial of levodopa. During the following two years and six months, he developed a tendency to lean backwards with frequent falling and his mobility gradually worsened to a point where he could only walk with the assistance of two people. There was further cognitive deterioration with episodic confusion and hallucinations. He developed a dressing apraxia, visuospatial defect, Broca's type dysphasia and severe dysarthria. He died of bronchopneumonia three years after the onset of his illness.

\section{Pathological examination}

At necropsy he was found to have bilateral lower lobe bronchopneumonia and a right pulmonary artery embolus. The fixed brain weighed $1300 \mathrm{~g}$ and there was generalised gyral atrophy and bilateral pallor of the substantia nigra and the locus coeruleus. Blocks were taken from representative cerebral, brainstem and cerebellar areas. The sections were stained with haematoxylin and eosin, luxol fast blue/cresyl violet,. Bielschowsky silver impregnation and anti-ubiquitin
Case History with urinary frequency for two years and recently associated with incontinence; a cystometrogram was compatible with an unstable bladder. In addition, he gave a six month history of generalised slowing of movement, falls, failing memory, a soft voice and difficulty in swallowing. He was seen by two of the authors (DJB and RSJF) who found a supranuclear ophthalmoplegia with poor voluntary and pursuit movements in both vertical and horizontal directions and intact doll's eye movements. He also had severe axial
This 71 year old man was initially admitted immunostain. Neurofibrillary tangles and neuritic plaques (demonstrated by Bielschowsky silver method) and Lewy bodies (antiubiquitin immuno-stain) were counted in seven micron sections of the hippocampus, frontal and temporal cortex.

In each cortical region a rectangular area of 7-12 $\mathrm{mm}^{2}$ was outlined and counted using an eyepiece graticule with parallel sweeps of the microscope stage. There were flame shaped tangles, mature neuritic plaques and Lewy bodies in the cerebral cortex (fig la and table). Tangles were confined to the allocortex and 
Figure 1a Cortical Lewy bodies in two neurons of layer $V$ of the temporal cortex, Ubiquitin $\times 150$ $1 b$ Brainstem Lewy bodies in the paramedian pontine reticular formation, haematoxylin and eosin, $\times 700$.

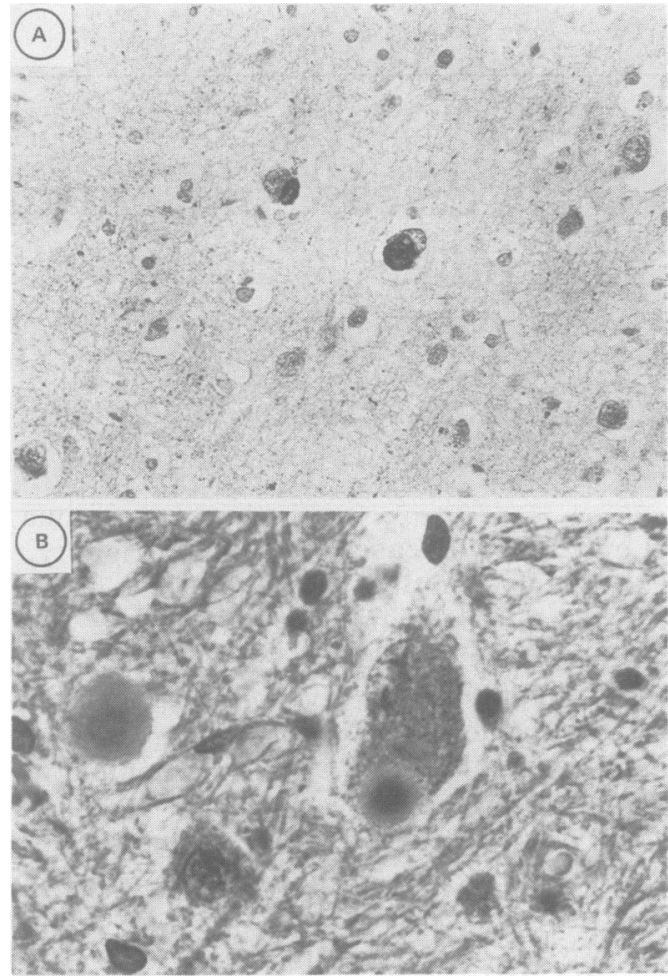

Table Neurofibrillary tangle, Lewy body and neuritic plaque counts (number per $\mathrm{mm}^{2}$ )

\begin{tabular}{|c|c|c|c|}
\hline Region & Tangle & Lewy body & Plaque \\
\hline Frontal & 0 & $3 \cdot 3$ & $8 \cdot 5$ \\
\hline & 0 & $2 \cdot 0$ & 14.9 \\
\hline Temporal T1 & 0 & 3.9 & $31 \cdot 1$ \\
\hline $\mathrm{T} 2$ & 0 & $1 \cdot 1$ & $4 \cdot 8$ \\
\hline T3 & 0 & $2 \cdot 8$ & $5 \cdot 7$ \\
\hline Hippocampus & $7 \cdot 3$ & $2 \cdot 1$ & $14 \cdot 8$ \\
\hline Subiculum & $5 \cdot 0$ & 1.4 & $9 \cdot 2$ \\
\hline Entorhinal & $9 \cdot 1$ & $2 \cdot 8$ & $13 \cdot 1$ \\
\hline
\end{tabular}

Lewy bodies were more widely distributed including the neocortex with a predilection for the temporal gyri and the deeper cortical layers. Neuritic plaque counts fulfilled Khachaturian's diagnostic criteria for Alzheimer's disease. ${ }^{6}$ There was a correlation between the numbers of neuritic plaques and Lewy bodies $(\mathrm{r}=0.85, \mathrm{p}<0.01)$, but not between neuritic plaques and tangles. The nucleus basalis of Meynert was considerably atrophic with severe neuronal loss and there was also nerve cell loss, free pigment and gliosis in the substantia nigra, locus coeruleus and dorsal vagal motor nucleus. The distribution of cell loss in the substantia nigra was typical of Parkinson's disease being greatest in the caudal ventrolateral cell groups. ${ }^{7}$ Tangles were absent in the nucleus basalis of Meynert, but were present in the brainstem nuclei although less frequently than Lewy bodies, 20 times less in the substantia nigra and four times less in the locus coeruleus. In the dorsal vagal motor nucleus, one neuron was found which contained both inclusions, (fig 2). Lewy bodies were found in the pontine paramedian reticular formation, (fig 1b), and tangles and neuritic plaques were found in the periaqueductal grey matter. The IIIrd, IVth and VIth nerve nuclei, the interstitial nucleus of Cajal, the rostral interstitial nucleus of the

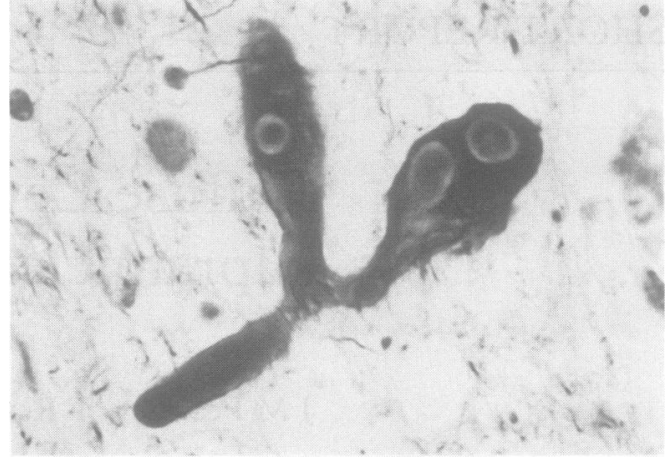

Figure 2 Co-existent Lewy bodies and neurofibrillary tangle neuron of the dorsal-vagal motor nucleus, Bielschowsky, $\times 1000$.

medial longitudinal fasiculus and the nucleus of Darkschewitsch appeared normal with no intraneuronal inclusions. Other areas typically involved in SRO were normal.

\section{Discussion}

The presence of a supranuclear gaze palsy, axial rigidity, early dementia and failure to respond to levodopa led to the diagnosis of SRO in this patient. Clinically, there was a suggestion that the dementia was predominantly of a temporoparietal type, which would not be in keeping with this diagnosis. ${ }^{8}$ However, neuropsychological testing revealed global cognitive dysfunction with marked impairment of tests sensitive to frontal lobe dysfunction. Testing, as with SRO, was hindered by the ophthalmoplegia. As the disease progressed the dementia became more severe than that usually seen in SRO.

The cornerstone in the diagnosis of SRO is the presence of a supranuclear gaze palsy, but this has also been described in a number of other multisystem degenerations including one case of DLBD.$^{89}$ Furthermore, a transient supranuclear palsy associated with infection has been described in two cases of Parkinsons's disease $^{10}$ and pathological changes have been found in nuclei involved in the control of eye movements: nucleus of Darkschewitsch, rostral interstitial nucleus of the medial longitudinal fasciculus, interstitial nucleus of Cajal and periaqueductal grey matter. ${ }^{11}$

In our case the prominent findings were neuritic plaques and NFTs in the periaqueductal grey matter and Lewy bodies in the pontine paramedian reticular formation. In this limited study we were unable to come to a conclusion as to the pathological basis of the ophthalmoplegia. Possible responsible sites could be the frontal eye fields, periaqueductal grey matter (vertical gaze) and pontine paramedian reticular formation (horizontal gaze). It has been suggested that lesions of the rostral interstitial nucleus of the medial longitudinal fasiculus cause a vertical gaze palsy and whether it is up or down is determined by the medio-lateral extent of the lesion. ${ }^{12}$

It has also been shown that a lesion of the interstitial nucleus of Cajal may cause a vertical gaze palsy and nuchal dystonia in exten- 
sion in cats. ${ }^{13}$ The gaze palsy, however, may be secondary to destroying fibres from the rostral interstitial nucleus of the medial longitudinal fasciculus passing through the interstitial nucleus of Cajal.

Antibody was provided by Professor Brian Anderton, Institute of Psychiatry, London.

1 Yoshimura M. Cortical changes in the Parkinsonian brain: a contribution to the delineation of 'diffuse Lewy body disease'. $J$ Neurol 1983;229:17-33.

2 Gibb WRG, Esiri MM, Lees AJ. Clinical and pathological features of diffuse cortical Lewy body disease (Lewy body dementia). Brain 1987;110:1131-54.

3 Lennox G, Lowe J, Landon M, Byrne EJ, Mayer RJ, Godwin-Austin RB. Diffuse Lewy body disease: correlative neuropathology using anti-ubiquitin immunocytochemistry. J Neurol Neurosurg Psychiatry 1989;

4 Steele JC, Richardson JC, Olszewski J. Progressive supra- nuclear palsy. Arch Neurol 1964;10:333-59.

5 Kristensen MO. Progressive supranuclear palsy-20 years later. Acta Neurol Scand 1985;71:177-89.

6 Khachaturian ZS. Diagnosis of Alzheimer's disease. Arch Neurol 1985;42:1097-105.

7 Hassler R. Zur Pathologie der Paralysis Agitans und des Postenzephalitischen Parkinsonismus. J Psychol Neurol 1938;48:387-476.

8 Lees AJ. The Steele-Richardson-Olszewski syndrome (progressive supranuclear palsy). In: Marsden CD, Fahn S, eds. Movement disorders 2, London: Butterworth
1987;272-87.

9 Lewis AJ, Gawel MJ. Diffuse Lewy body disease with dementia and oculomotor dysfunction. Movement disorders 1990;5:143-7.

10 Guiloff RJ, George RJ, Marsden CD. Reversible supranuclear ophthalmoplegia associated with parkinsonism. $J$ Neurol Neurosug Psychiatry 1980;43:552-4.

11 Hunter SB, Guy JR, Gainesville H. Neuropathological correlation of eye movement abnormalities in idiopathic Parkinson's disease. Annals Neurol 1985;18:136-7.

12 Buttner-Ennever JA, Buttner U, Cohen B, Baugartner G. Vertical gaze paralysis and the rostral interstitial nucleus of the medial longitudinal fasiculus. Brain 1982;105: $125-49$.

13 Fukushima-Kudo J, Fukushima K, Tashiro K. Rigidity and dorsiflexion of the neck in progressive supranuclear palsy dorsiflexion of the neck in progressive supranuclear palsy
and the interstitial nucleus of Cajal. $J$ Neurol Neurosurg Psychiatry 1987;50:1197-203. 